\title{
Sarcoma-180 tumor affects the quality of oocytes in mice
}

\author{
ZIHANG CHEN, SIMIN WANG, XUEXIA LUO and YANHONG YANG \\ The First Affiliated Hospital (School of Clinical Medicine), Guangdong Pharmaceutical University, \\ Guangzhou, Guangdong 510080, P.R. China
}

Received May 19, 2020; Accepted December 8, 2020

DOI: $10.3892 / \mathrm{ol} .2021 .12442$

\begin{abstract}
Numerous factors can affect the quality of oocytes; however, the effects of cancer on the quality of oocytes and the underlying mechanisms remain unclear. In the present study, the effects of the sarcoma-180 (S-180) cell line on the quality of oocytes were investigated using S-180 tumor-bearing mice. In total, 42 female $\mathrm{C} 57 \mathrm{BL} / 6 \mathrm{~J}$ mice were randomly divided into the tumor-bearing group and the control group, with 21 mice per group. The weight of the mice and ovaries were recorded, and blood glucose, serum insulin, lipopolysaccharide, triglyceride (TG) and total cholesterol (TC) levels were analyzed using the corresponding detection kits. Hematoxylin and eosin staining was performed to observe the pathological changes of the ovarian tissue, and reverse transcription-quantitative PCR (RT-qPCR) was used to analyze the expression levels of meiosis arrest female 1 (MARF1), SUMO-specific protease 7 (SENP7), aralkylamine N-acetyltransferase (AANAT), cell division cycle $25 \mathrm{~B}$ and glycine-rich protein 3 . The results of the present study revealed that the number of oocytes in the two groups of mice was similar; however, the number of abnormal oocytes was increased in the tumor-bearing group. The serum levels of TG and TC were significantly elevated in the tumor-bearing group compared with in the control group $(\mathrm{P}<0.01)$. Additionally, RT-qPCR analysis demonstrated that the expression levels of SENP7 were downregulated, while the expression levels of MARF1 and AANAT were upregulated in the ovaries of the tumor-bearing group compared with in the control group $(\mathrm{P}<0.01)$. In conclusion, the findings of the present study suggested that cancer may affect the reproductive system of mice and decrease the quality of oocytes by regulating the expression levels of reproduction-associated genes. These results provided novel insights into the reproductive ability of patients with cancer.
\end{abstract}

Correspondence to: Dr Yanhong Yang, The First Affiliated Hospital (School of Clinical Medicine), Guangdong Pharmaceutical University, 19 Nong-Lin-Xia Road, Yue-Xiu, Guangzhou, Guangdong 510080, P.R. China

E-mail: 1764941457@qq.com

Key words: Sarcoma-180, tumor-bearing mice, oocyte, quality

\section{Introduction}

Cancer is the second largest disease after cardiovascular disease in terms of morbidity and mortality, and has become the second leading cause of death in humans (1). Additionally, cancer has been reported to affect other systems in the body; for example, it was discovered that rats implanted with Yoshida ascites sarcoma rapidly developed anemia (2). In addition, the metastasis of colorectal cancer to the liver exacerbated skeletal muscle wasting by regulating differential gene expression signatures (3). On the other hand, abnormalities in the whole body have been discovered to affect the progression of cancer. For example, a previous study identified a strong association between the prevalence of autism and the incidence of breast cancer in situ (4). Diabetic patients with insulin resistance were identified to exhibit changes in gene expression levels that increased the risk of colorectal cancer (5). Therefore, the state of the cancer may affect the reproductive system and the quality of the oocytes.

Successful embryo and fetal development are dependent on the quality of the oocytes, which is regulated by various factors (6), for instance, the appropriate metabolism and sufficient ATP production were discovered to be associated with the quality of oocytes and the preimplantation embryo development (6). Alves et al (7) reported that short-term supplementation with a high-fat diet altered the dynamics of follicles, but did not affect the proportion of morphologically normal oocytes. Meanwhile, Yu et al (8) identified that increased levels of reactive oxygen species were present in the ovary with increased age, and the subsequent oxidative stress led to a decrease in the number and quality of oocytes. Mihalas et al (9) suggested that proteasome dysfunction may be the primary reason for the decline in the quality of age-related oocytes. Additionally, it was reported that melatonin enhanced DNA repair, thereby preventing the quality of oocytes from decreasing during meiosis (10). In addition, clinical medicines can affect the quantity and quality of oocytes; a previous study demonstrated that common first-line anti-tuberculosis drugs, including rifampicin, isoniazid, pyrazinamide, streptomycin and ethambutol, decreased the number of oocytes in females and increased their degeneration, as well as increasing the rate and changing the distribution pattern of cytoplasmic organelles (11).

With changes to modern lifestyle habits and the deterioration of the environment, the age of cancer onset is decreasing $(12,13)$; however, it is currently unknown whether 
the reproductive system of female patients with non-reproductive system cancers is affected. In the present study, the effects of cancer on the quantity and quality of oocytes were studied using sarcoma-180 (S-180) tumor-bearing mice, which may provide novel insights into the reproductive ability following cancer.

\section{Materials and methods}

Cell culture. The mouse S-180 cell line was purchased from the Cell Bank of Type Culture Collection of the Chinese Academy of Sciences. Cells were cultured in RPMI-1640 medium (Gibco; Thermo Fisher Scientific, Inc.) supplemented with $10 \%$ FBS (HyClone; Cytiva) and maintained at $37^{\circ} \mathrm{C}$ in a humidified atmosphere containing $5 \% \mathrm{CO}_{2}$.

Animal studies and tissue collection. The experimental procedures in the present study were approved by the Committee on Laboratory Animal Care and Use of Guangdong Pharmaceutical University (Guangzhou, China). In total, 42 C57BL/6J female mice (age, 5-8 weeks; weight, 13 $\pm 2.0 \mathrm{~g}$ ) were purchased from Hunan SJA Laboratory Animal Co., Ltd. The mice were housed in a temperature-controlled room $\left(23 \pm 2^{\circ} \mathrm{C}\right)$ at a relative humidity of $50 \pm 10 \%$ with a 12 -h light/dark cycle and free access to a standard diet and water. The general conditions of the mice were checked every day. The mice were randomly divided into 2 groups: The tumor-bearing group and the control group, each containing 21 mice. The S-180 cells were washed with pre-chilled PBS (HyClone; Cytiva) and the density of the cells within the fluid was subsequently adjusted to $1 \times 10^{7}$ cells $/ \mathrm{ml}$ prior to $0.2 \mathrm{ml}$ injected into the right armpit of the mice in the tumor-bearing group to establish the S-180 tumor-bearing model. The control group was subcutaneously inoculated with an equal volume of PBS buffer solution at the same location. The mice were checked every day for $\sim 10$ days, and the tumors could be seen or felt under the skin of the right armpit of the tumor-bearing group. The allowed maximum diameter of the tumors was $1.5 \mathrm{~cm}$, and the allowed maximum tumor volume was $2,000 \mathrm{~mm}^{3}$. The formula used to calculate tumor volume was $\mathrm{V}\left(\mathrm{mm}^{3}\right)=\pi / 6 \mathrm{ab}^{2}$ with $\mathrm{a}>\mathrm{b}$ (14). The average tumor volume of the tumor-bearing group was $1,740 \pm 490 \mathrm{~mm}^{3}$. The images of tumors are shown in Fig. S1.

Inducing superovulation in mice. On the 10th day after cell injection, each mouse was intraperitoneally injected with 5 IU pregnant horse serum gonadotropin (PMSG; Shanghai Gaochuang Chemical Technology Co., Ltd.) and after 48 h, the mice were intraperitoneally injected with 5 IU human chorionic gonadotropin (HCG; Shanghai Gaochuang Chemical Technology Co., Ltd.). After injection of HCG for $12 \mathrm{~h}$, the mice were sacrificed by cervical dislocation to collect the bilateral ovaries. The quality of the ovaries was assessed using an electronic scale (precision $0.1 \mathrm{mg}$; Beijing Taize Jiaye Technology Development Co., Ltd.). The bilateral oviducts were separated and placed in PBS at room temperature. The ampulla of the oviduct was punctured with a $26 \mathrm{G}$ needle, and the oocytes were removed with a pipette and transferred into M2 culture medium containing hyaluronidase (Shanghai Yuanye Biotechnology Co., Ltd.) and the cumulus cells were subsequently removed. The morphology of the oocytes could be clearly observed when the granular cells alongside the oocytes had disappeared. Briefly, the oocytes were transferred into the M2 culture using the pipette and washed 3 times in the nutrient solution. The total number of oocytes was counted, and the morphological characteristics of the oocytes under different magnification were observed using a Leica DMi8 light microscope (Leica Microsystems, Inc.) under x100, x200 and x400 magnification.

Serum profile assays. The mice were adequately anesthetized with ether for blood collection, and the pinch reflex was monitored to ensure full anesthesia. The anesthesia method was approved by the Committee on Laboratory Animal Care and Use of Guangdong Pharmaceutical University. The whole blood was collected from the orbit, and after collecting $1 \mathrm{ml}$ blood, the mice were sacrificed by cervical dislocation. The blood glucose levels were detected using a blood glucose detection kit (cat. no. 561510) purchased from Shanghai Rongsheng Biology Pharmaceutical Co., Ltd., according to the manufacturer's protocol. Serum was obtained from the blood by centrifugation at $1,160 \mathrm{x} g$ at $4^{\circ} \mathrm{C}$ for $15 \mathrm{~min}$. The concentrations of serum total cholesterol (TC), triglyceride (TG), insulin and lipopolysaccharide (LPS) were analyzed using the TC detection kit (cat. no. A111-1-1), TG detection kit (cat. no. A110-1-1), insulin detection kit (cat. no. Z26018441) and LPS detection kit (cat. no. Z24018439), respectively, according to the manufacturers' protocols. The detection kits for analyzing TG and TC levels were purchased from Nanjing Jiancheng Bioengineering Institute. The ELISA kits for analyzing insulin and LPS levels were purchased from Wuhan Huamei Biological Engineering Co., Ltd.

Hematoxylin and eosin $(H \& E)$ staining. The ovarian tissues were fixed in $4 \%$ paraformaldehyde overnight at $4^{\circ} \mathrm{C}$, dehydrated with an ascending alcohol series and embedded into paraffin. Paraffin-embedded tissues were subsequently sliced into $4-\mu \mathrm{m}$-thick sections and stained with hematoxylin (Sigma-Aldrich; Merck KGaA) for 3 min and eosin (Sigma-Aldrich; Merck KGaA) for $20 \mathrm{sec}$, both at room temperature. The stained sections were visualized using an automated quantitative pathology imaging system (PerkinElmer, Inc.).

Reverse transcription-quantitative PCR (RT-qPCR). Total RNA was extracted from the native ovarian tissue of the mice using TRIzol ${ }^{\circledR}$ reagent (Invitrogen; Thermo Fisher Scientific, Inc.). Total RNA was reverse transcribed into cDNA using a PrimeScript RT Reagent kit (Takara Bio, Inc.) according to the manufacturer's protocol. The primers were designed using the National Center for Biotechnology Information (NCBI) online primer design software (https:/www.ncbi.nlm.nih. gov/tools/primer-blast/index.cgi?LINK_LOC=BlastHome) according to the gene sequence published on the NCBI Gene webpage, and synthesized by Sangon Biotech Co., Ltd. The specific primer sequences are presented in Table I. RT-qPCR was subsequently performed using a SYBR Premix Ex Taq kit (Takara Bio, Inc.) and a PikoReal Real-Time PCR system (Thermo Fisher Scientific, Inc.). The following thermocycling conditions were used for qPCR: $95^{\circ} \mathrm{C}$ for $30 \mathrm{sec}$, followed by 40 cycles of $95^{\circ} \mathrm{C}$ for $5 \mathrm{sec}, 60^{\circ} \mathrm{C}$ for $20 \mathrm{sec}$ and $65^{\circ} \mathrm{C}$ for 
A
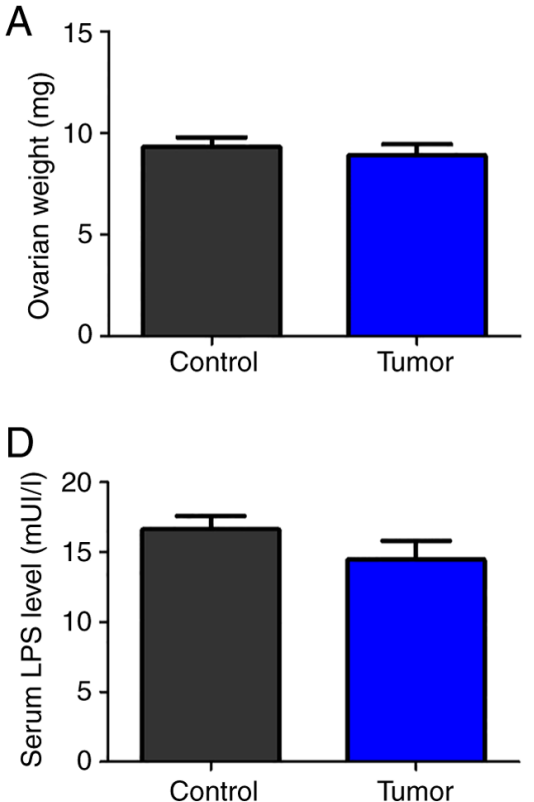

$\mathrm{B}$

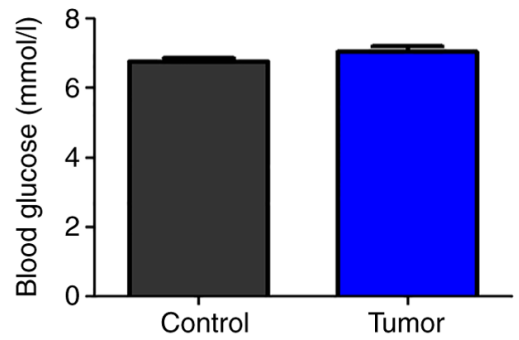

$E$

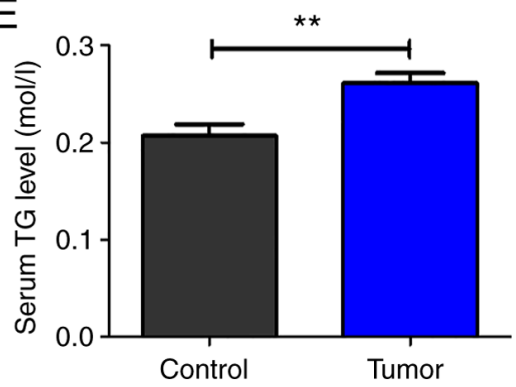

C

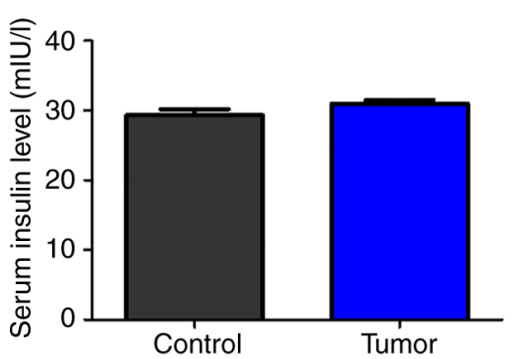

$\mathrm{F}$

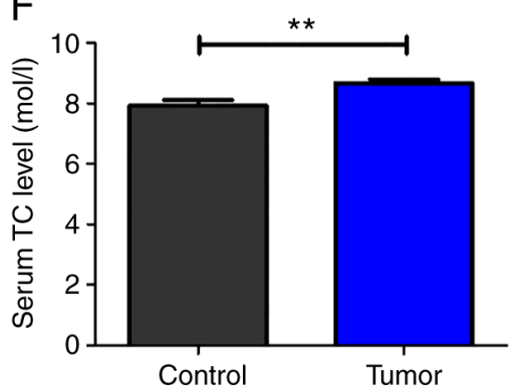

Figure 1. Ovary weight and serum analysis. (A) Ovary weight. Levels of (B) blood glucose, (C) serum insulin, (D) serum LPS, (E) serum TG and (F) serum TC. ${ }^{* *} \mathrm{P}<0.01$. LPS, lipopolysaccharide; TG, triglyceride; TC, total cholesterol.

$15 \mathrm{sec}$. The data were analyzed using the $2^{-\Delta \Delta \mathrm{Cq}}$ method (15), and GAPDH was used as the internal loading control.

Statistical analysis. Statistical analysis was performed using SPSS 24.0 software (IBM Corp.) and all data are expressed as the mean \pm SD. Unpaired Student's t-test was used to determine the statistical differences between two groups. The differences in the abnormal rate of oocytes between the different groups were analyzed using a $\chi^{2}$ test. Each experiment was repeated $\geq 3$ times. $\mathrm{P}<0.05$ was considered to indicate a statistically significant difference.

\section{Results}

Effects of S-180 tumors on the serum profile. No significant difference was identified in the weight of the ovaries between the tumor-bearing and the control groups (Fig. 1A). Similarly, no significant differences were observed in the levels of blood glucose, serum insulin and LPS between the two groups (Fig. 1B-D). However, the serum TG and TC levels were significantly increased in the tumor-bearing group compared with in the control group (Fig. 1E and F).

Effects of S-180 tumors on the morphology of the ovaries and oocytes. The results of the $\mathrm{H} \& \mathrm{E}$ staining revealed that there were more mature oocytes ready to be expelled at the edge of the ovary in the control group, which were accompanied by a large number of granulosa cells around the follicles (Fig. 2A and B). In the tumor-bearing group, there were fewer surrounding granulosa cells and the majority of the follicles were in the primary phase (Fig. 2C and D). Each oocyte was separated by a glass needle under the microscope, and the morphology of the oocytes was observed. A qualified mature oocyte was suitable in size $(80-100 \mu \mathrm{m})$, with a complete membrane and nucleus, and a full cytoplasm; the first polar body had also been expelled (Fig. 3). An abnormal oocyte was
Table I. Primer sequences of genes for reverse transcription-quantitative PCR.

\begin{tabular}{ll}
\hline Genes & \multicolumn{1}{c}{ Sequences } \\
\hline MARF1 & F: 5'-TCAGAACTCCAGCTCCGAACAGG-3' \\
& R: 5'-GGATGGCAGGCATGAAGGAACAG-3' \\
SENP7 & F: 5'-TTGTTTATCCTCCACCACCTAC-3' \\
& R: 5'-CTCTGAGCCACTGATAGATCTG-3' \\
AANAT & F: 5'-CTTTATCTCTGTCTCCGGTACC-3' \\
& R: 5'-CTGAGTAAGTCTCTCCTTGTCC-3' \\
CDC25B & F: 5'-GGACCACGAATGTGCCGCTTCA-3' \\
& R: 5'-AGTTCCCTCCTGCTCCGTTCCC-3' \\
GPR3 & F: 5'-CTCACCAGAGATGAGCTTGATG-3' \\
& R: 5'-TAAGTGAGAGCATTGTACAGGG-3' \\
GAPDH & F: 5'-GCATCCACTGGTGCTGCC-3' \\
& R: 5'-TCATCATACTTGGCAGGTTTC-3' \\
\hline
\end{tabular}

$\mathrm{F}$, forward; R, reverse; MARF1, meiosis arrest female 1; SENP7, SUMO-specific protease 7; AANAT, aralkylamine $\mathrm{N}$-acetyltransferase; CDC25B, cell division cycle 25B; GPR3, glycine-rich protein 3 .

found to be either enlarged or shrunk in size, with a broken cell membrane, a dissolved nucleus and a broken or retained polar body (Fig. 3). The number of normal and abnormal oocytes was counted; the results revealed that there were 39 abnormal oocytes out of a total of 93 oocytes in the control group, which was higher than that of a previous study (16), while there were 36 abnormal oocytes out of a total of 63 oocytes in the tumor-bearing group (Table II). The abnormal rate of oocytes in the tumor-bearing group $(57.10 \%)$ was increased compared with that in the control group (41.90\%) (Table II). 
Table II. Number of oocytes and abnormal rate.

\begin{tabular}{lcccr}
\hline Groups & Total oocytes & Abnormal oocytes & $\chi^{2}$ & P-value \\
\hline Control group & 93 & 39 & 3.479 & 0.062 \\
Tumor-bearing group & 63 & 36 & & \\
\hline
\end{tabular}
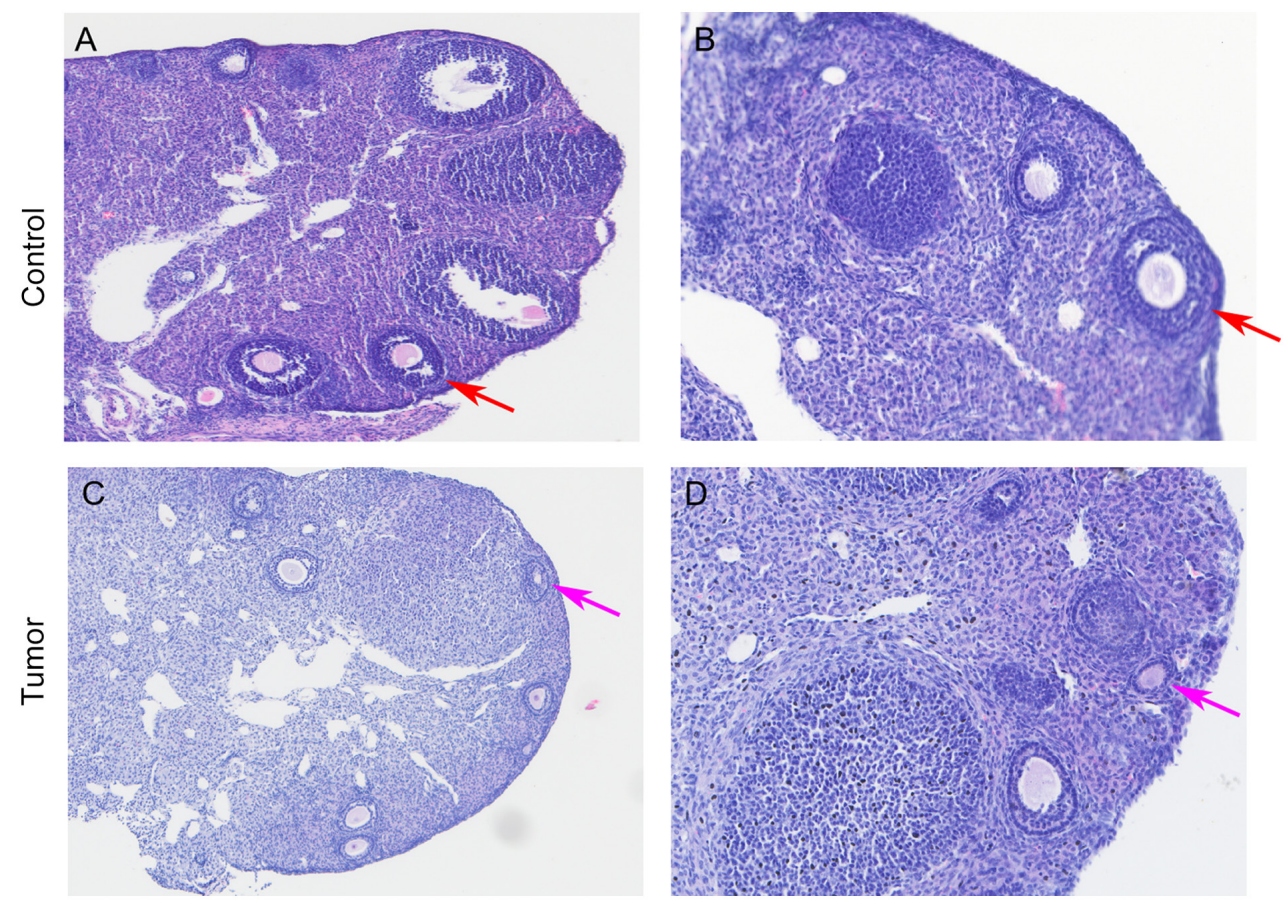

Figure 2. Hematoxylin and eosin staining of ovarian tissues. Ovary of the control group at (A) x100 and (B) x200 magnification. Ovary of the tumor-bearing group at (C) x100 and (D) x200 magnification. The red arrow indicates a mature follicle; the pink arrow indicates an abnormal follicle.
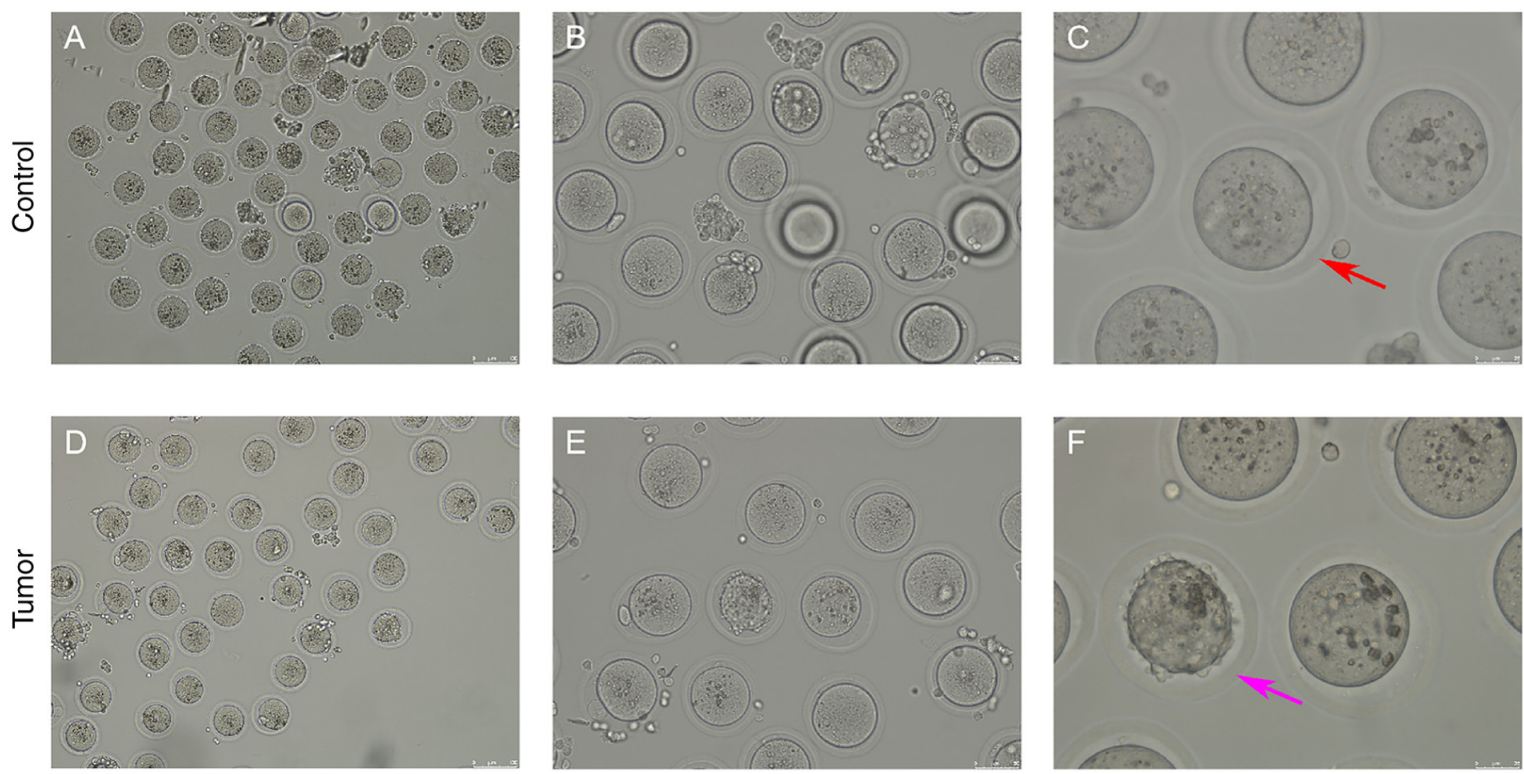

Figure 3. Observation of the morphology of oocytes. Oocytes of the control group at (A) x100, (B) x200 and (C) x400 magnification. Oocytes of the tumor-bearing group at (D) x100, (E) x200 and (F) x400 magnification. The red arrow indicates a normal oocyte; the pink arrow indicates an abnormal oocyte.

$S$-180 tumors regulate the expression levels of reproduction-associated genes. The expression levels of reproduction-associated genes were further analyzed in the ovarian tissue of the mice. The results of the RT-qPCR 

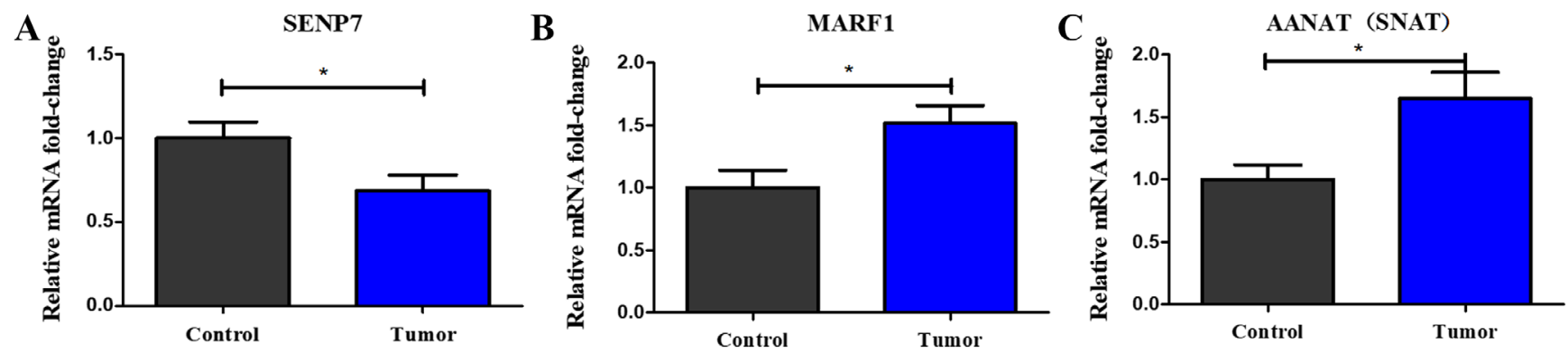

D

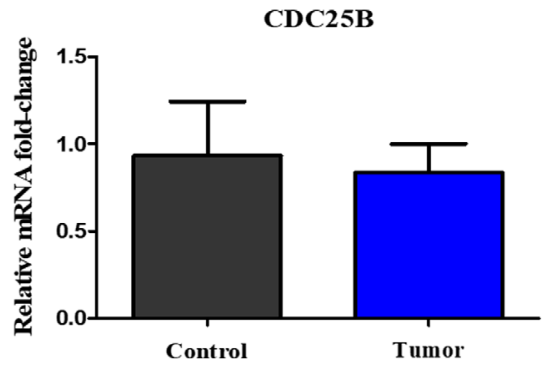

$\mathbf{E}$

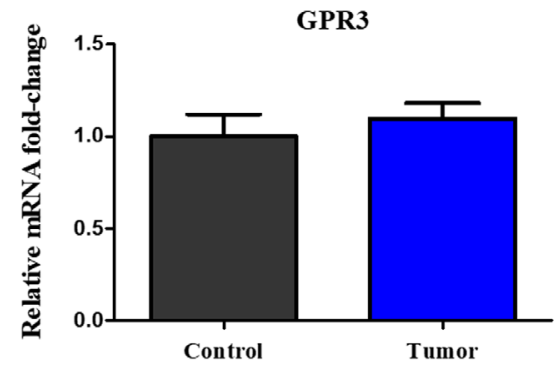

Figure 4. Expression levels of reproduction-associated genes in ovaries analyzed by reverse transcription-quantitative PCR. Expression levels of (A) SENP7, (B) MARF1, (C) AANAT, (D) CDC25B and (E) GRP3. 'P<0.05. SENP7, SUMO-specific protease 7; MARF1, meiosis arrest female 1; AANAT, aralkylamine $\mathrm{N}$-acetyltransferase; CDC25B, cell division cycle 25B; GRP3, glycine-rich protein 3.

analysis revealed that the expression levels of SUMO-specific protease 7 (SENP7) were significantly downregulated in the tumor-bearing group compared with those in the control group (Fig. 4A), while the expression levels of meiosis arrest female 1 (MARF1) and aralkylamine $\mathrm{N}$-acetyltransferase (AANAT) were significantly upregulated in the tumor-bearing group compared with those in the control group (Fig. 4B and C). There were no significant differences identified in the expression levels of cell division cycle 25B (CDC25B) and glycine-rich protein 3 (GRP3) between the two groups (Fig. 4D and E).

\section{Discussion}

In the present study, the effects of S-180 tumor on the quantity and quality of oocytes were investigated using tumor-bearing mice. The results revealed that the serum TG and TC levels were significantly increased in the tumor-bearing group compared with in the control group, alongside the number of abnormal oocytes. The abnormal rate of oocytes of the tumor-bearing group (57.10\%) was increased compared with that of the control group $(41.90 \%)$, although the number of abnormal oocytes in the control group was higher than that in a previous report (39 abnormal oocytes out of 93 oocytes) (16), which may be associated with the batch deference of mice. It was previously reported that the growth and survival of tumor cells depended on lipid metabolism, as the lipids could provide the energy for the proliferation of tumor cells (17). Moreover, Wang et al (18) reported that the levels of TG, TC and low-density lipoprotein were negatively associated with the number of normal fertilized oocytes, and that the TG levels were negatively associated with the number of oocytes and cleavage embryos. The results of the present study identified that the serum TG and TC levels were significantly increased in the tumor-bearing group, which may explain how the S-180 tumor may affect the quantity and quality of the oocytes.
However, whether serum TG and TC levels are associated with the progression of cancer and the oocyte quality in patients with cancer requires further investigation.

The expression levels of reproduction-associated genes were further analyzed in the ovaries of mice using RT-qPCR. The results demonstrated that the expression levels of SENP7 were significantly downregulated in the tumor-bearing group, while the expression levels of MARF1 and AANAT were significantly upregulated in the tumor-bearing group compared with in the control group. SUMOylation can regulate numerous different cellular processes, and SENP7 is a de-SUMOylation enzyme (19). It was reported that SENP7 was essential for chromatin relaxation in response to DNA damage, and the de-SUMOylation by SENP7 provided a permissive chromatin environment for DNA repair (20). Since SENP7 is essential for heterochromatin integrity and DNA repair, its roles in the mammalian reproductive system were studied. Huang et al (21) reported that the deficiency of SENP7 in oocytes arrested the meiosis process at the prophase I and metaphase I stages, resulting in a substantial decrease in the number of mature oocytes in mice. In addition, the maternal-zygotic transition was defective in SENP7-depleted embryos, which led to very little blastocyst production (21). These findings suggested that SENP7 may be essential in the epigenetic programming of the oocyte and embryonic development. The present results demonstrated that the expression levels of SENP7 were significantly downregulated in the tumor-bearing group compared with in the control group, which was consistent with the findings of Huang et al (21), suggesting that S-180 tumors may affect the quantity and quality of oocytes by regulating the expression levels of SENP7.

MARF1 is essential for the control of meiosis and the maintenance of genomic integrity during the development of oocytes, and mutations in MARF1 were discovered to upregulate a cohort of transcripts and increase retrotransposon 
expression, leading to defective cytoplasmic maturation, meiotic arrest and female infertility (22-24). AANAT is expressed in oocytes at all stages of follicular development and it synthesizes serotonin to melatonin enzymatically, together with acetylserotonin O-methyltransferase (25). The synthesis of melatonin in oocytes is essential for the maturation of oocytes and the development of the embryo through its ability to protect the oocytes from oxidative damage $(25,26)$. In the present study, the expression levels of MARF1 and AANAT were significantly upregulated in the tumor-bearing group compared with in the control group. These effects may be due to the S-180 tumors creating a high stress environment that affected the function of the ovaries in the mice, which in turn upregulated the expression levels of several reproduction-associated genes, such as MARF1 and AANAT, as a protective mechanism. However, these mechanisms require further study.

$\mathrm{CDC} 25 \mathrm{~B}$ is an essential regulator for meiotic resumption in mouse oocytes (27), and GPR3 potentiates meiotic competence by increasing cyclic AMP levels (28). The present results identified no significant differences in the expression levels of CDC25B and GRP3 between the tumor-bearing and control groups.

In conclusion, the findings of the present study revealed that the number of oocytes in the tumor-bearing group and the control group of mice was similar, while the number of abnormal oocytes was increased in the tumor-bearing group. The serum levels of TG and TC were significantly elevated in the tumor-bearing group compared with in the control group. In addition, the expression levels of SENP7 were downregulated, while the expression levels of MARF1 and AANAT were upregulated in the ovaries of the tumor-bearing group compared with the control group. To the best of our knowledge, the present study was the first to investigate the possible effects of non-reproductive system tumors on the female reproductive system, in addition to suggesting the possible mechanisms of the effects of S-180 tumors on oocytes in mice. These results may provide the foundations to use for the clinical reference of female patients with cancer when determining their fertility; however, the specific mechanisms remain unclear, and changes in the expression levels of other reproduction-associated genes require to be verified.

\section{Acknowledgements}

Not applicable.

\section{Funding}

The present study was supported by the National Natural Science Foundation of China (grant no. 81803912), the Scientific Research Project of the Administration of Traditional Chinese Medicine of Guangdong Province (grant no. 20182079), the Characteristic Innovation Project (Natural Science) of the Education Department of Guangdong Province and the 'Innovation Strong School Project' of Guangdong Pharmaceutical University (grant no. 2017KTSCX102), the Science and Technology Project of Yue-Xiu District of Guangzhou (grant no. 2018-WS-011) and the Innovation and Entrepreneurship Training Program for College Students of Guangdong Province (grant no. S201910573021).

\section{Availability of data and materials}

The datasets used and/or analyzed during the current study are available from the corresponding author on reasonable request.

\section{Authors' contributions}

YY designed and conceived the study, wrote the first draft of the manuscript and critically revised the manuscript. ZC established the mouse model and performed the reverse transcription-quantitative PCR. ZC, SW and XL performed the serum analysis and the H\&E staining. All authors read and approved the final manuscript.

\section{Ethics approval and consent to participate}

The experimental procedures in the present study were approved by the Committee on Laboratory Animal Care and Use of Guangdong Pharmaceutical University (Guangzhou, China).

\section{Patient consent for publication}

Not applicable.

\section{Competing interests}

The authors declare that they have no competing interests.

\section{References}

1. Global Burden of Disease Cancer Collaboration; Fitzmaurice C, Allen C, Barber RM, Barregard L, Bhutta ZA, Brenner H, Dicker DJ, Chimed-Orchir O, Dandona R, et al: Global, regional, and national cancer incidence, mortality, years of life lost, years lived with disability, and disability-adjusted life-years for 32 cancer groups, 1990 to 2015: A systematic analysis for the global burden of disease study. JAMA Oncol 3: 524-548, 2017.

2. Bhanushali AA, Raghunathan R, Kalraiya RD and Mehta NG: Cancer-related anemia in a rat model: Alpha2-macroglobulin from Yoshida sarcoma shortens erythrocyte survival. Eur J Haematol 68: 42-48, 2015.

3. Huot JR, Novinger LJ, Pin F, Narasimhan A, Zimmers TA, O'Connell TM and Bonetto A: Formation of colorectal liver metastases induces musculoskeletal and metabolic abnormalities consistent with exacerbated cachexia. JCI Insight 5: 136687, 2020.

4. Kao HT, Buka SL, Kelsey KT, Gruber DF and Porton B: The correlation between rates of cancer and autism: An exploratory ecological investigation. PLoS One 5: e9372, 2010.

5. Xia Z, Su YR, Petersen P, Qi L, Kim AE, Figueiredo JC, Lin Y, Nan H, Sakoda LC, Albanes D, et al: Functional informed genome-wide interaction analysis of body mass index, diabetes and colorectal cancer risk. Cancer Med 9: 3563-3573, 2020.

6. Dunning KR and Robker RL: Promoting lipid utilization with 1-carnitine to improve oocyte quality. Anim Reprod Sci 134: 69-75, 2012.

7. Alves JPM, Fernandes CCL, Rossetto R, Silva CPD Galvão ITOM, Bertolini M and Rondina D: Impact of short nutrient stimuli with different energy source on follicle dynamics and quality of oocyte from hormonally stimulated goats. Reprod Domest Anim 54: 1206-1216, 2019.

8. Yu S, Zhao Y, Feng Y, Zhang H, Li L, Shen W, Zhao M and Min L: $\beta$-carotene improves oocyte development and maturation under oxidative stress in vitro. in vitro Cell Dev Biol Anim 55: 548-558, 2019.

9. Mihalas BP, Bromfield EG, Sutherland JM, De Iuliis GN, McLaughlin EA, Aitken RJ and Nixon B: Oxidative damage in naturally aged mouse oocytes is exacerbated by dysregulation of proteasomal activity. J Biol Chem 293: 18944-18964, 2018. 
10. Leem J, Bai GY, Kim JS and Oh JS: Melatonin protects mouse oocytes from DNA damage by enhancing nonhomologous end-joining repair. J Pineal Res 67: e12603, 2019.

11. Rao A, Nayak G, Kumari S, Kalthur SG, Mutalik SP, Mutalik S, Adiga SK and Kalthur G: Exposure to first line anti-tuberculosis drugs in prepubertal age reduces the quality and functional competence of spermatozoa and oocytes in Swiss albino mice. Environ Toxicol Pharmacol 73: 103292, 2020.

12. Patel SG and Ahnen DJ: Colorectal cancer in the young. Curr Gastroenterol Rep 20: 15, 2018.

13. Moore $\mathrm{K}$ and Brewer MA: Endometrial cancer: Is this a new disease? Am Soc Clin Oncol Educ Book 37: 435-442, 2017.

14. Raab M, Kappel S, Krämer A, Sanhaji M, Matthess Y, Kurunci-Csacsko E, Calzada-Wack J, Rathkolb B, Rozman J, Adler T, et al: Toxicity modelling of Plk1-targeted therapies in genetically engineered mice and cultured primary mammalian cells. Nat Commun 2: 395, 2011.

15. Livak KJ and Schmittgen TD: Analysis of relative gene expression data using real-time quantitative PCR and the 2(-Delta Delta C(T)) method. Methods 25: 402-408, 2001.

16. He YT, Yang LL, Zhao Y, Shen W, Yin S and Sun QY Fenoxaprop-ethyl affects mouse oocyte quality and the underlying mechanisms. Pest Manag Sci 75: 844-851, 2019.

17. Brantley KD, Riis AH, Erichsen R, Thorlacius-Ussing O, Møller HJ and Lash TL: The association of serum lipid levels with colorectal cancer recurrence. Cancer Epidemiol 66: 101725, 2020.

18. Wang S, Wang J, Jiang Y and Jiang W: Association between blood lipid level and embryo quality during in vitro fertilization. Medicine (Baltimore) 99: e19665, 2020.

19. Xiang JW, Xiao Y, Gan Y, Chen H, Liu Y, Wang L, Nie Q, Liu F, Gong X, Fu JL, et al: Glucose oxidase- and UVA-induced changes in the expression patterns of seven de-sumoylation enzymes (SENPs) are associated with cataract development. Curr Mol Med 19: 48-53, 2019.

20. Garvin AJ, Densham RM, Blair-Reid SA, Pratt KM, Stone HR, Weekes D, Lawrence KJ and Morris JR: The deSUMOylase SENP7 promotes chromatin relaxation for homologous recombination DNA repair. EMBO Rep 14: 975-983, 2013.
21. Huang CJ, Wu D, Jiao XF, Khan FA, Xiong CL, Liu XM, Yang J, Yin TL and Huo LJ: Maternal SENP7 programs meiosis architecture and embryo survival in mouse. Biochim Biophys Acta Mol Cell Res 1864: 1195-1206, 2017.

22. Su YQ, Sugiura K, Sun F, Pendola JK, Cox GA, Handel MA, Schimenti JC and Eppig JJ: MARF1 regulates essential oogenic processes in mice. Science 335: 1496-1499, 2012.

23. Cao GY, Li MZ, Wang H, Shi LY and Su YQ: Interference with the C-terminal structure of MARF1 causes defective oocyte meiotic division and female infertility in mice. J Biomed Res 32: 58-67, 2018.

24. Yao Q, Cao G, Li M, Wu B, Zhang X, Zhang T, Guo J, Yin H, Shi L, Chen J, et al: Ribonuclease activity of MARF1 controls oocyte RNA homeostasis and genome integrity in mice. Proc Natl Acad Sci USA 115: 11250-11255, 2018.

25. Sakaguchi K, Itoh MT, Takahashi N, Tarumi W and Ishizuka B: The rat oocyte synthesises melatonin. Reprod Fertil Dev 25: 674-682, 2013.

26. Tamura H, Jozaki M, Tanabe M, Shirafuta Y, Mihara Y, Shinagawa M, Tamura I, Maekawa R, Sato S, Taketani T, et al: Importance of melatonin in assisted reproductive technology and ovarian aging. Int J Mol Sci 21: 1135, 2020.

27. Kang H, Hwang SC, Park YS and Oh JS: Cdc25B phosphatase participates in maintaining metaphase II arrest in mouse oocytes. Mol Cells 35: 514-518, 2013.

28. Firmani LD, Uliasz TF and Mehlmann LM: The switch from cAMP-independent to cAMP-dependent arrest of meiotic prophase is associated with coordinated GPR3 and CDK1 expression in mouse oocytes. Dev Biol 434: 196-205, 2018.

This work is licensed under a Creative Commons Attribution-NonCommercial-NoDerivatives 4.0 International (CC BY-NC-ND 4.0) License. 